\title{
La importancia de la pragmática en la traducción de textos sobre nuevas identidades de género
}

\author{
ANTONIO Jesús MARTÍNEZ \\ Pleguezuelos \\ Universidad Complutense de \\ Madrid \\ Departamento de Estudios \\ Románicos, Franceses, Italianos y \\ Traducción \\ C/ del Profesor Aranguren 2, \\ 28040 Madrid \\ E-mail: antmar17@ucm.es
}

\author{
AleXandra Santamaría URBIETa \\ Universidad de Alcalá \\ Departamento de Filologia \\ Moderna \\ Colegio de Caracciolos \\ C/ Trinidad 3 \\ 28801 Alcalá de Henares \\ E-mail: \\ alexandra.santamaria@uah.es
}

THE IMPORTANCE OF PRAGMATICS IN THE TRANSLATION OF TEXTS ON NEW GENDER IDENTITIES

RESUMEN: El objetivo de este estudio es analizar los retos que supone la traducción del inglés al español de un texto sobre reasignación de sexo por la complejidad que ciertos aspectos de género, así como la terminología y fraseología relacionada con ello, implican para la constitución de una nueva identidad. Realizaremos nuestro análisis siguiendo una metodologia cualitativa a partir de las soluciones aportadas por estudiantes de tercer curso de la asignatura de traducción científico-técnica del Grado en Lenguas Modernas y Traducción de la Universidad de Alcalá. Los resultados muestran que, aunque existe cierto nivel de concienciación entre los estudiantes a la hora de encontrar soluciones de traducción a los problemas planteados, es necesario hacer un mayor hincapié en la formación en traducción desde un enfoque pragmático. Esto contribuirá a que sean conscientes del potencial de su trabajo a la hora de construir y difundir conocimiento a través del texto traducido.

PALABRAS ClAVES: identidad, género, traducción, formación, enfoque pragmático.

SUMARIO: 1. Introducción. 2 . Contexto académico. 3. Metodología. 4. Resultados. 5. Conclusiones.
ABSTRACT: The aim of this study is to analyse the challenges involved in the translation from English to Spanish of a text about sex reassignment due to the complexity that certain aspects of gender, as well as terminology and phraseology related to it, imply for the constitution of a new identity. For the analysis, a qualitative methodology will be followed based on the solutions provided by thirdyear students of the ScientificTechnical Translation course of the Degree in Modern Languages and Translation from the University of Alcalá. The results show that, although there is a certain level of awareness among students when it comes to finding translation solutions in this type of texts, greater emphasis should be placed on translation training from a pragmatic approach. This will help them to be aware of the potential of their work when it comes to building and disseminating knowledge through the translated text.

KEY WORDS: identity, gender, translation, training, pragmatic approach.

SUMMARY: 1 . Introduction. 2. Academic context. 3. Methodology. 4. Results. 5. Conclusions.

\author{
Elena Alcalde Peñalver \\ Universidad de Alcalá \\ Departamento de Filologia \\ Moderna \\ Colegio de Caracciolos \\ C/ Trinidad 3 \\ 28801 Alcalá de Henares \\ E-mail: e.alcalde@uah.es
}

\section{L'IMPORTANCE DE LA PRAGMATIQUE DANS LA TRADUCTION DE TEXTES SUR LES NOUVELLES IDENTITES DE GENRE}

RÉSUMÉ: L'objectif de cette étude est d'analyser les difficultés liées à la traduction de l'anglais vers l'espagnol d'un texte sur l'opération de réattribution sexuelle en raison de la complexité que certains aspects du genre, ainsi que la terminologie et la phraséologie, impliquent pour la constitution d'une nouvelle identité. Pour l'analyse, une méthodologie qualitative construite à partir des solutions apportées par les étudiants de la troisième année du cours de traduction scientifique et technique des études en Langues Modernes et Traduction de l'Université d'Alcalá. Les résultats montrent que, même si les étudiants sont particulièrement sensibilisés à la recherche de solutions de traduction dans ce type de textes, il convient de donner plus d'importance à la formation en traduction selon une approche pragmatique. Cela les aidera à prendre conscience du potentiel de leur travail en matière de construction et de diffusion des connaissances grâce au texte traduit.

MOTS CLÉS: identité, genre, traduction, formation, approche pragmatique.

SOMMAIRE: 1. Introduction. 2. Contexte académique. 3 . Méthodologie. 4. Résultats. 5. Conclusions.
Fecha de Recepción Fecha de Revisión Fecha de Aceptación Fecha de Publicación 


\section{La importancia de la pragmática en la traducción de textos sobre nuevas identidades de género}

Antonio Jesús Martínez Pleguezuelos, Alexandra Santamaría Urbieta \& Elena Alcalde PEÑALVER

\section{INTRODUCCIÓN: HACIA NUEVOS CONCEPTOS INTERDISCIPLINARES DE TRADUCCIÓN}

Tal y como postulan los enfoques más recientes en los estudios de traducción, resulta cada vez más complicado pensar y concebir la traducción como una disciplina estanca e independiente. Muy al contrario, la permeabilidad de esta disciplina es palmaria si observamos con detenimiento el trabajo cotidiano de los traductores y la necesidad de buscar en otros terrenos académicos y profesionales afines estrategias y herramientas desde las que desarrollar su labor. Tal y como ya recordaba Hickey (1998: 1) en la introducción de la antología The Pragmatics of Translation, el trabajo del traductor va más allá de simplemente decir en otra lengua lo que aparece en el mensaje original:

Whatever translation is in its entirety, it seems to involve semiotic, linguistic, textual, lexical, social, sociological, cultural and psychological aspects or elements, all of which are being studied nowadays as determining factors in whatever the translator does.

La evolución hacia nuevas fronteras académicas desde los estudios de traducción ha sido estable y cada vez más visible durante las últimas tres décadas en este campo. De este modo, la introducción de nuevas metodologias, de nuevos enfoques críticos para abordar soluciones de traducción, de diferentes visiones en torno al concepto de fidelidad en la traducción o de concebir la lealtad al autor del original desde distintas perspectivas ha enriquecido en gran medida el debate en el seno mismo de la traducción, alcanzando cotas de complejidad y dinamismo nunca antes observadas.

En una extensión de estas premisas e inspirado sobre la hipótesis de que el fenómeno traductológico no tiene por qué inscribirse en un único campo, la propuesta académica de Gentzler (2017) erigida sobre el concepto de "post-translation studies", retomado a su vez de Nergaard y Arduini (2011), aboga por el acercamiento, e incluso solapamiento, de los estudios de traducción con otras áreas académicas. Ya sea desde los estudios feministas, el ámbito postcolonial, la sociolingüística, la pragmática, la crítica literaria o la lingüística, por citar solo algunas de las disciplinas de larga tradición incorporadas en los estudios de traducción, parece evidente que en muchas ocasiones resulta más interesante aproximarnos al estudio de lo que tiene lugar en los puntos de encuentro de diversas áreas, en zonas discursivas de conflicto en las que, forzosamente, será necesario recurrir a andamiajes teóricos localizados más allá de la traducción como vía para reescribir el trabajo que nos ocupa. 
En este mismo sentido, de forma paralela a la perspectiva post-traductológica de Gentzler, el “outward turn" que desarrolla Bassnett (2017: viii-ix) reafirma la multidisciplinariedad hacia la que las nuevas investigaciones en traducción caminan con paso firme. De hecho, afirma esta autora que aún en la actualidad no se ha alcanzado un consenso definitivo sobre lo que constituyen los estudios de traducción (Bassnett 2017: viii) y anima a una reestructuración y redefinición del ámbito que no suponga un punto final para los estudios de traducción, sino un punto y seguido hacia la "expansion of its self- imposed boundaries, so that the field can reach out to other disciplines and become more open to ideas about translational issues coming from researchers who may not be primarily engaged in translation" (Bassnett 2017: ix).

Sin ningún atisbo de duda, existe un interés creciente por parte de muchos autores por la incorporación de la pragmática como vía de estudio de la comunicación intercultural en la traducción. En lineas generales, un enfoque pragmático en traducción nos ayudará a comprender el "translation procedure, process and product from the point of view of what is (potentially) done by the original author in or by the text, what is (potentially) done in the translation as a response to the original, [and] how and why it is done in that way in that context" (Hickey 1998: 4). El texto, por si mismo, no nos ofrece toda esta información si no lo consideramos en un contexto específico, entendiendo este último no solo como el entorno inmediato en una comunicación, sino como un entramado de condicionantes presentes, pasados y futuros que determinan la comunicacion: "expectations about the future, scientific hypotheses or religious beliefs, anecdotal memories, general cultural assumptions, beliefs about the mental state of the speaker, may all play a role in interpretation" (Sperber y Wilson 1986: 15).

Ya en 1955 Austin exponia en How to Do Things with Words que cuando empleamos el lenguaje estamos poniendo en marcha una serie de acciones capaces de construir conocimiento y significado a través de actos de habla que actúan en un contexto dado. Dichos actos de habla comunican la información que desea trasmitir el emisor, pero, al mismo tiempo, cargan con todo un sistema de valores, creencias y conocimiento presupuesto compartido entre los participantes que tendrán un papel muy activo en la comunicación. Esta capacidad para generar significado ha pasado a ser protagonista desde que Lefevere y Bassnett (1990) incorporaran el giro cultural en los estudios de traducción, y aún entre las investigaciones de mayor impacto en la actualidad, continúa siendo un aspecto central para muchos investigadores en traducción por entender esta actividad como un elemento constitutivo de la realidad a nuestro alrededor, además de "the fundamental process that allows the very existence of any signifying activity" (Gentzler 2012: $\mathrm{s} / \mathrm{p})$. Del mismo modo, Cronin (2006: 1) en su ya canónico Translation and Identity localiza el ejercicio de la traducción en "the centre of any attempt to think about questions of identity in human society", aspecto sobre el que volveremos en nuestro análisis por la complejidad de ciertos fragmentos en la 
traducción y por el potencial que contiene la traducción para moldear nuevas identidades para nuevos grupos culturales y lingüisticos. Huelga decir, por otra parte, que en los casos de textos con un contenido delicado que aborda aspectos que inciden de manera directa en la constitución de la identidad de un colectivo, como el texto seleccionado para este análisis (Anexo), el traductor deberá prestar mucha atención al modo en que reescribe dicha identidad, pero también qué premisas, valores y prejuicios afloran en las sociedades de cultura y de llegada y cómo influirán en su trabajo.

En este sentido, es necesario señalar que, desde un punto de vista pragmático, es obligatorio considerar que en toda traducción se emplea un uso del lenguaje interpretativo (Gutt 1998: 46) para restablecer un contenido desde un mensaje original y que, en muchos casos, los contextos en los que se produce el texto original y posteriormente la traducción son completamente diferentes, tanto en el plano espacial como en el temporal, por lo que "it can be very difficult to individuate a shared cognitive environment to use as the basis for pragmatic analysis" (Dicerto 2018: 39). En el caso de los textos escritos, como el que nos ocupa en la presente investigación, Cooren (2008) mantiene que el significado original que plasma el autor se encuentra supeditado al contexto en el cual el lector se aproxima al texto, por lo que en buena medida depende de él. Con ello, el enfoque pragmático no solo resulta útil, sino necesario para establecer los limites conceptuales en los que la comunicación tiene lugar. Por otra parte, como comprobaremos mediante algunos ejemplos seleccionados para nuestro trabajo, las presuposiciones adquieren en la práctica traductora una gran relevancia, dependientes siempre del contexto en el que se produce la reescritura. Sin embargo, hemos de recordar que, a pesar de la preponderancia de las presuposiciones a la que aludimos, su estudio en los procesos de traducción se ha pasado por alto durante mucho tiempo y no se ha profundizado lo suficiente en este aspecto (Fawcett 1998: 115).

En función de estas premisas, consideramos necesario tener en cuenta, antes de continuar con el siguiente apartado, la intencionalidad del texto y las consecuencias que pueden acarrear distintos problemas en la traducción. Como veremos detalladamente, en el texto que presentamos sobre reasignación de sexo, traducido como encargo en una clase de traducción, si prestamos atención a la máxima de la cortesía politeness que autores como Brown y Levinson (1987) añaden a las máximas de Grice (1975), una alteración en el género al hacer referencia a los pacientes puede alterar de forma muy negativa la imagen que se proyecta de estos a través de la traducción. Retomando el sentido amplio e interdisciplinar de los estudios de traducción con el que comenzábamos estas páginas, si combinamos, como muestra, un tratamiento apropiado del género de los pacientes sometidos a una reasignación de sexo con los diversos enfoques teóricos adoptados desde las corrientes feministas y los estudios LGTBIQ+, la traducción queda expuesta como terreno fértil desde el que construir conocimiento y representar nuevas identidades a través de las 
reescrituras llevadas a cabo. De ahí que, como hemos postulado y como veremos con distintos ejemplos más adelante, consideremos urgente expandir el concepto de traducción en el sentido que planteamos en estas páginas.

\section{ConteXto ACADÉmico}

Este estudio se ha realizado como parte de la asignatura de traducción especializada (científico-técnica) del tercer curso del Grado en Lenguas Modernas y Traducción de la Universidad de Alcalá. Se trata de la primera asignatura en la que el alumnado de este programa de estudios se enfrenta a la traducción de textos especializados. En la clase había un total de veinte alumnos y realizaron la traducción tras haber trabajado durante cuatro semanas con textos médicos. En cuanto a la cuestión de género, no habian recibido formación previa ni indicaciones en clase, por lo que para nuestro análisis partiamos de una muestra de estudiantes que no contaba con una concienciación específica sobre cómo debían traducir aspectos de esta índole. El texto objeto de estudio constituyó un encargo individual que era parte de la evaluación continua de la asignatura y suponía un $20 \%$ de la nota final. Se pidió además a los alumnos que realizaran un comentario sobre los problemas de traducción que habían encontrado y cómo los habian resuelto.

\section{Metodología}

Para este estudio hemos seguido una metodología de análisis cualitativo utilizada en un estudio previo realizado por Roskoka y Rupniece (2016). Los autores seguian un análisis en base a unidades de contenido, agrupadas por categorias analiticas. De esta forma, los resultados analizados los mostramos en tablas específicas para cada categoria, de acuerdo con el modelo de literatura teórica de Geske y Grinfelds (2006). Es necesario señalar que la metodología cualitativa permite comprender los fenómenos educativos y transformar la realidad social existente (Savenye y Robinson, 2005). Esto contribuye a su vez a la elaboración de procesos de mejora en la enseñanza y aprendizaje (Svensson y Doumas, 2013), ya que, como es el caso de este estudio, nos permite analizar las elecciones terminológicas, fraseológicas y de género de nuestros estudiantes y comprender el por qué de sus decisiones a la hora de realizar su traducción.

Para nuestro análisis hemos tenido en cuenta tres categorias principales: una específica de género, otra terminológica y otra fraseológica. No obstante, estas dos últimas categorias tienen también un componente de género en el que basamos la elección de las unidades de nuestro análisis, ya que, tal y como indicamos en la introducción, su traducción incide de manera directa en la constitución de la identidad de un colectivo, por lo que el traductor deberá prestar especial atención al modo en el que reescribe dicha identidad. 
En cuanto al género, es necesario especificar que el texto incluye recomendaciones tras una operación de reasignación de sexo, en este caso de masculino a femenino y, aunque en el texto original no resulta necesario especificar el uso de pronombres masculino o femenino para referirse al público al que va dirigido, sí que lo es en español e incluso realizar la concordancia con algunos adjetivos. Por lo tanto, en esta categoría analizaremos el número de estudiantes que fueron conscientes de estos cambios y sus comentarios al respecto. Además, incluiremos en esta categoría el análisis del término nursing, para observar igualmente el uso del género masculino o femenino en la traducción del término, que cuenta con un uso predominante como "enfermera" en español, en comparación con la denominación de la mayoría de las profesiones en este idioma cuyo uso en masculino genérico cuenta con una mayor aceptación. Del mismo modo, en el texto aparecía el nombre de la doctora responsable (Dr. Bowers), que accediendo a la web del encargo de traducción se podía comprobar que era de género femenino. Analizaremos igualmente si los alumnos se encargaron de realizar esta comprobación o si lo dejaron en masculino en español. Esto nos permitirá observar si mantienen un estereotipo de género a través del uso del masculino o femenino en su traducción (Garcés García y Filardo Llamas, 2004).

En relación con la categoría de terminologia, tal y como indica Corbacho Sánchez (2005: 67), “a la hora de abordar la cuestión terminológica, nos encontramos con inusitada frecuencia que una multitud de designaciones se usan indistintamente según el criterio de un determinado especialista o por desconocimiento entre algunos conceptos". En esta categoria hemos analizado la traducción de cis-gender y killer-orgasm. No hemos analizado otros términos de carácter más especializado como drain tube o Foley catheter al no contener implicaciones de género de interés para nuestro estudio.

En cuanto a la fraseología, hemos seleccionado dos unidades para nuestro análisis. Se trata, por un lado, de "earlier friskiness is posible anecdotically" y, por otro, "the much coveted B-spot, which is the direct counterpart of the natal female "G-spot". Siguiendo de nuevo las indicaciones de Corbacho Sánchez (2005), una característica esencial para la traducción de una unidad fraseológica es la idiomaticidad. En este sentido, Corpas Pastor (1997: 26) indica que un fraseologismo es idiomático cuando "el significado global de dicha unidad no es deducible del significado aislado de cada uno de sus elementos constitutivos". Para ello, indica tres grados de equivalencia (Corpas Pastor 2000: 490-491):

- Equivalencia plena. Se produce cuando hay una correspondencia total entre una unidad fraseológica en un idioma y otro, con un mismo significado denotativo y connotativo, una misma base metafórica y frecuencia de uso.

Equivalencia parcial. Las unidades presentan divergencias en la base metafórica, el plano léxico-semántico y sintáctico, la frecuencia de uso y las condiciones socioculturales.

- Equivalencia nula. No hay semejanza entre la unidad fraseológica en una lengua y otra. 
En el siguiente apartado mostramos y analizamos las soluciones de traducción adoptadas por los alumnos y los comentarios más significativos que realizaron al respecto.

\section{Resultados}

En esta primera categoría de análisis nos centramos en cómo los estudiantes han abordado la traducción de cuestiones de género en el texto.

\begin{tabular}{|l|c|}
\hline Categoria de análisis: GÉNERO & Unidades de contenido \\
\hline Uso del masculino o femenino & Siete estudiantes optaron por la opción de traducir el \\
& texto utilizando fórmulas en femenino: \\
& Our patients: nuestras pacientes \\
& Everyone heals at different pace: Cada una se recu- \\
& pera a su ritmo \\
& An expectation of each and every patient: Una expec- \\
& tativa de todas y cada una de nuestras pacientes \\
\hline
\end{tabular}

Tabla 1: Opciones de traducción relativas al género

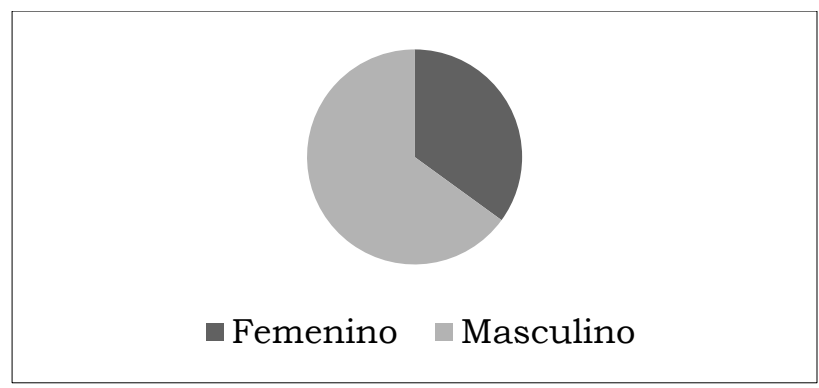

Figura 1: Adopción en español de fórmulas en femenino

A continuación se incluyen los comentarios que realizaron para justificar su decisión (aparecen en inglés y español según la elección del estudiante, ya que ambas se consideran lenguas de trabajo en la asignatura):

- He puesto todo lo referido a las pacientes en femenino porque la persona que lea esto ya se habrá operado y se trata de una operación de género de hombre a mujer.

- Since the target readership of the text are men who do not identify with their birth-assigned gender and seek medical assistance to transition to the female gender, I have decided to use the feminine form of the word.

- En la lengua inglesa no hay un género tan marcado en los sustantivos ni en ciertas profesiones. Sin embargo, en la lengua española sí, y he optado por usar el plural en femenino debido a la naturaleza de la operación y a quién va dirigida. 
- Me planteé el hecho de tratar a las pacientes en femenino o masculino genérico pero, tras considerar el contexto (cirugía de reasignación de género, mujer transexual), opté por utilizar el femenino genérico.

- Adjectives have been translated in feminine to not misgender anyone.

- The decision to keep the pronouns of the whole text as feminine stems from a desire to appeal to the trans women who, being one of the most likely people to get the surgery, would be reading this hoping not to get misgendered. I believe that this text should not be written or translated using masculine pronouns, because generally trans women will prefer to use she/her pronouns.

- Creo que es muy importante tener en cuenta el poder del lenguaje. Es muy probable que este documento sea el primero que lean estas mujeres, que acaban de empezar a sentirse completas, y por eso considero que es imprescindible considerar la influencia del lenguaje, es decir, que ellas vean que en este texto las palabras se están refiriendo a ellas y reafirman su feminidad, lo que podría tener un efecto importante en su autoestima.

En cuanto a las profesiones, diez estudiantes optaron por la traducción the nursing como enfermera (ocho en singular y dos en plural), tres en masculino y siete una opción neutra (la enfermería o personal de enfermería).

\begin{tabular}{|l|l|}
\hline Categoría de análisis: GÉNERO & \multicolumn{1}{|c|}{ Unidades de contenido } \\
\hline Nursing & Una enfermera (8) \\
& Las enfermeras (2) \\
& Los enfermeros (3) \\
& La enfermeria (3) \\
& Personal de enfermería (4) \\
\hline
\end{tabular}

Tabla 2: Traducción del término nursing

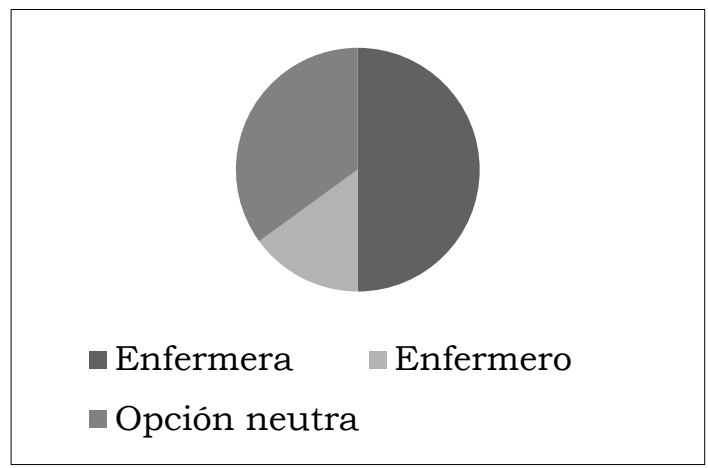

Figura 2: Traducción del término nursing

Finalmente, doce estudiantes no comprobaron que Dr. Bowers se trataba de una mujer.

\begin{tabular}{|l|l|}
\hline Categoria de análisis: GÉNERO & \multicolumn{1}{|c|}{ Unidades de contenido } \\
\hline Dr. Bowers & Dra. Bowers (8) \\
\hline
\end{tabular}


Como se puede observar, más de la mitad del alumnado de la clase no han sido conscientes de las cuestiones de género en la traducción al español aun tratándose de un texto sobre el tema. No obstante, es interesante señalar cómo un alto porcentaje de la clase sí que se detuvo a reflexionar al respecto y muestran concienciación sobre el estereotipo de género al que hacian referencia Garcés García y Filardo Llamas (2004) y que puede existir a través del lenguaje, lo que han reflejado en sus comentarios. En estos casos, un mayor hincapié en la formación de los estudiantes desde un enfoque pragmático para la traducción de este tipo de textos habria contribuido a comprender, tal y como indicaba Hickey (1998), la necesidad de tener en cuenta el contexto específico en el que se enmarca el texto para evitar que el sistema de valores, creencias y conocimiento presupuesto transmitido a través de la sociedad influya en las soluciones de traducción adoptadas. Como hemos indicado en la introducción, una alteración en el género al hacer referencia a los receptores del texto meta repercute de forma negativa en la imagen que se proyecta de la construcción de su nueva identidad, por lo que es necesario generar una mayor concienciación sobre las opciones de traducción que debemos adoptar para que esto no ocurra.

En la segunda categoría de análisis terminológico, estos fueron los resultados obtenidos:

\begin{tabular}{|l|l|}
\hline Categoria de análisis: TERMINOLOGÍA & \multicolumn{1}{|c|}{ Unidades de contenido } \\
\hline Cis-gender female & Mujer cisgénero (18 respuestas) \\
& $\begin{array}{l}\text { Mujer no transexual } \\
\text { Género femenino }\end{array}$ \\
\hline
\end{tabular}

Tabla 4: Traducción de cis-gender female

El alumno que optó por "mujer no transexual" justificó su decisión diciendo que el término en español "cisgénero" aún se utiliza poco y buscó una opción que le permitiera expresar la idea de una forma clara y precisa. En efecto, aunque el término aún no esté recogido en la RAE, su uso cada vez está más popularizado. De hecho, la revista Time dedicó un artículo en 2014 a la explicación de su significado en inglés:

Cisgender is a word that applies to the vast majority of people, describing a person who is not transgender. If a doctor announces, "It's a girl!" in the delivery room based on the child's body and that baby grows up to identify as a woman, that person is cisgender.

En otro artículo de esta misma revista publicado en 2017 también se aportaba una definición del término: Someone whose gender is in line with the sex they were assigned at birth. 
En español, lo vemos explicado en diversos blogs y publicaciones sobre LGTB. En el periódico La Vanguardia, Atienza (2017) lo define como el término que describe a aquellas personas que se identifican con el género con el que nacieron. Es decir, lo contrario a las personas transgénero. Ambas palabras provienen del latín: el prefijo "cis" significa "de este lado", mientras que "trans" es "del otro lado". En esta misma linea se encuentra la definición del glosario del género de National Geographic en español (2018).

En cuanto al último término objeto de nuestro análisis, estas fueron las diversas soluciones de traducción encontradas:

\begin{tabular}{|l|l|}
\hline Categoría de análisis: TERMINOLOGÍA & \multicolumn{1}{|c|}{ Unidades de contenido } \\
\hline Killer orgasm & Orgasmo mortal (2) \\
& Orgasmo asesino \\
& Orgasmo de muerte (3) \\
Orgasmo de infarto (2) & \\
& Increible orgasmo \\
& Superorgasmo \\
& Orgasmo revolucionario \\
& Orgasmo salvaje \\
& Orgasmo explosivo \\
& Orgasmo perfecto (2) \\
& Gran orgasmo \\
& Un orgasmo tan increíble \\
& Magnifico orgasmo \\
& Orgasmo tan deseado \\
& Orgasmo que te lleve al cielo \\
\hline
\end{tabular}

Tabla 5: Traducción de killer orgasm

En siete ocasiones, las soluciones intentaban guardar ese paralelismo con killer al mantener "mortal", "asesino", "de muerte" o "de infarto", término relacionados con el fallecimiento o enfermedad. El resto de las opciones dejaban de lado ese componente metafórico e intentaban trasmitir la idea de algo fuera de lo normal con adjetivos como "explosivo" o "revolucionario", o paráfrasis como "tan deseado" o "que te lleve al cielo".

Si tenemos en cuenta lo señalado anteriormente por Corbacho Sánchez (2005), en el caso de nuestro estudio observamos homogeneidad a la hora de traducir un término de relativa novedad como es "cisgénero", pero multitud de designaciones para la traducción de killer orgasm, debido al desconocimiento del concepto asociado a este término.

Finalmente, en cuanto a la fraseología, estos fueron los resultados analizados en función del tipo de equivalencia conseguida:

\begin{tabular}{|c|l|}
\hline Categoría de análisis: FRASEOLOGÍA & \multicolumn{1}{c|}{ Unidades de contenido } \\
\hline Early friskiness is possible anecdotically & $\begin{array}{l}\text { PLENA (5) } \\
\text { Puede haber algún jugueteo esporádico antes. }\end{array}$ \\
& $\begin{array}{l}\text { Puede realizar algunos juegos sexuales de forma } \\
\text { puntual antes. }\end{array}$ \\
& $\begin{array}{l}\text { Es posible y de forma esporádica la realización de } \\
\text { juegos sexuales. }\end{array}$ \\
\hline
\end{tabular}




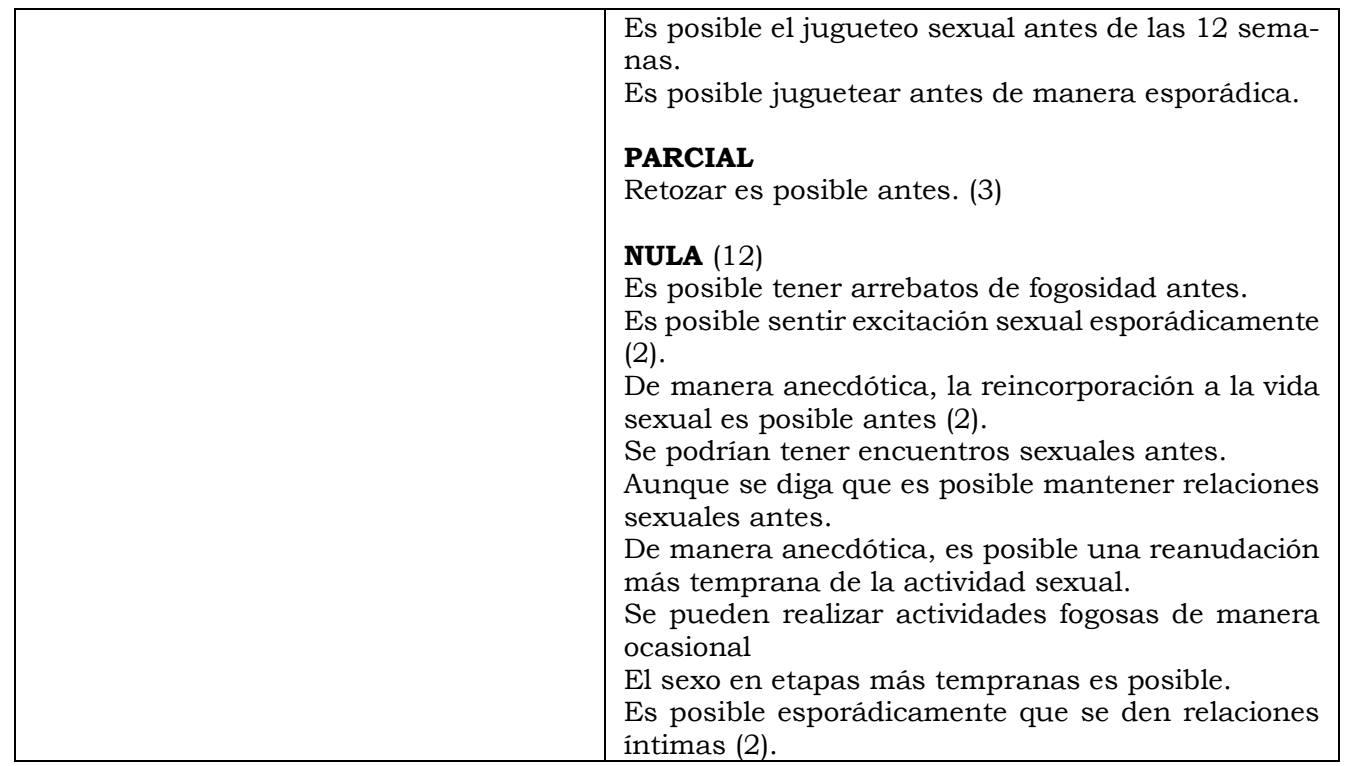

Tabla 6: Traducción de early friskiness is possible anecdoticallyz

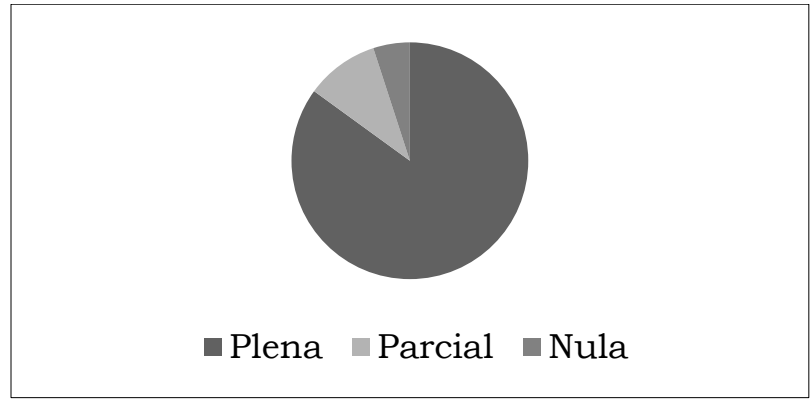

Figura 3: Tipos de equivalencia al traducir early friskiness is possible anecdotically

El término "friskiness" supuso un problema de traducción para todos los estudiantes. En el diccionario Merriam-Webster, este término se define como "playful" o "lively" y el diccionario Cambridge lo define como "the quality of liking to play or being full of activity". Por lo tanto, reanudar la actividad sexual y todas las opciones que impliquen mantener relaciones en su totalidad no serian expresiones equivalentes que funcionaran para su traducción. En cuanto a "retozar", según la RAE significa "realizar juegos eróticos con otra persona". Sin embargo, no es un verbo que encontrariamos normalmente en un texto publicado por una clínica sobre recomendaciones para personas que acaban de someterse a una cirugía. La opción de "jugueteo esporádico" sí funcionaría plenamente o "realizar algunos juegos sexuales". "Realizar actividades fogosas" transmite parcialmente la idea de "friskiness" pero le añade una carga de significado que no está presente en el término original. 


\begin{tabular}{|c|c|}
\hline Categoria de análisis: FRASEOLOGÍA & Unidades de contenido \\
\hline $\begin{array}{l}\text { Much coveted B-spot which is the direct } \\
\text { counterpart of the natal female G-spot }\end{array}$ & $\begin{array}{l}\text { PLENA (17) } \\
\text { El deseado punto B, el equivalente del punto G } \\
\text { que tienen las mujeres por nacimiento. } \\
\text { El tan codiciado punto B que equivale al punto } \\
\text { G de una mujer biológica. } \\
\text { El tan deseado punto B que es el equivalente del } \\
\text { punto G de una persona nacida mujer } \\
\text { El codiciado punto P, el equivalente del punto G } \\
\text { en la anatomía femenina. } \\
\text { El codiciado "punto B", que sería el equivalente } \\
\text { al punto G en las mujeres. (2) } \\
\text { El tan codiciado "punto B", que es el equivalente } \\
\text { del "punto G" femenino. (3) } \\
\text { El tan deseado “punto B", el equivalente exacto } \\
\text { al "punto G" de las personas nacidas con el sexo } \\
\text { femenino. } \\
\text { El ansiado "punto B", el equivalente del "punto } \\
\text { G" femenino de nacimiento. (2) } \\
\text { El tan ansiado "punto B", que es le equivalente } \\
\text { del "punto G" con el que nacen las mujeres. } \\
\text { El codiciado "punto B", el equivalente del "punto } \\
\text { G" femenino innato. } \\
\text { El deseado punto B, el equivalente del punto G } \\
\text { de una mujer cisgénero (2). } \\
\text { El deseado "punto B", que es el equivalente del } \\
\text { "punto G" femenino biologico. } \\
\text { PARCIAL (2) }\end{array}$ \\
\hline
\end{tabular}

Tabla 7: Traducción de much coveted B-spot which is the direct counterpart of the natal female G-spot

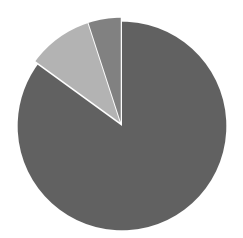

- Plena $\backsim$ Parcial $\backsim$ Nula

Figura 4: Tipos de equivalencia al traducir much coveted B-spot which is the direct counterpart of the natal female G-spot 
Por otro lado, como podemos observar, esta oración no supuso un problema de equivalencia para los estudiantes. No obstante, cabe destacar la variedad de opciones por las que optaron para traducir natal female. El estudiante que aportó la opción "nacidas con el sexo femenino" realizó el siguiente comentario: "Para evitar la posibilidad de herir sensibilidades de las personas que anhelan convertir su cuerpo en femenino, he neutralizado la expresión natal female y he optado en español por "las personas nacidas con el sexo femenino", en vez de "mujeres de nacimiento" o, mucho menos, "mujeres naturales".

En cuanto al término "B-spot", todos mantuvieron la B, aunque no sea una opción extendida aún en español. El estudiante que adoptó la opción "punto P", aportó el siguiente comentario al respecto:

I could not find an equivalent for G-spot (Gräfenberg Spot) and deduced that because of its position ("in front of the vaginal wall just beneath the bladder"), B-spot stemmed from "bladder". When consulting Transexualia, the official transsexuality webpage in Spain, I concluded that this spot has nothing to do with the bladder but the prostate gland, which is left intact in the surgery. It is possible then, that the term would make reference to this organ, and based on this it would be adequate to translate it as "punto P.

De nuevo en estos casos, aunque la búsqueda de equivalencias terminológicas y fraseológicas no supusiera un problema de traducción generalizado para la mayoria de los estudiantes, la formación previa desde un enfoque pragmático habría contribuido a establecer de forma más precisa los limites conceptuales en los que se enmarcaba el acto comunicativo del texto origen. Esto a su vez habria repercutido en que los estudiantes aportaran soluciones de traducción que transmitieran el mensaje del emisor de forma más precisa y adecuada al contexto específico del texto traducido.

\section{Conclusiones}

Este estudio nos ha permitido analizar las opciones de traducción adoptadas por estudiantes de una asignatura de traducción científico-técnica (inglés-español) del Grado en Lenguas Modernas y Traducción de la Universidad de Alcalá en materia de género, así como la terminología y fraseología relacionada con ello, en un texto sobre una operación de reasignación de sexo. A la hora de realizar la traducción, era muy importante tener en cuenta el contexto específico en el que se enmarcaba el acto comunicativo. No obstante, los alumnos no habian recibido ningún tipo de formación previa en la asignatura respecto a la temática de género del texto al que se enfrentaban. Esto habría contribuido a evitar que las presuposiciones, estereotipos y conocimientos previos que estos tenian repercutieran de forma negativa, a la hora de transmitir mediante su traducción a los receptores del texto meta, es decir, pacientes que se someten a este tipo de operación, el mensaje del emisor en cuanto a la construcción de su nueva identidad. Los resultados 
nos muestran que más de la mitad de los estudiantes no fueron conscientes de la traducción de cuestiones de género. No obstante, resultaron de gran interés las reflexiones que realizaron sobre los estereotipos existentes al respecto. La búsqueda de equivalentes a nivel terminológico no resultó un problema generalizado en este sentido, pero sí lo fue encontrar un equivalente pleno a nivel fraseológico para una de las unidades analizadas.

A la vista de estos resultados y desde el enfoque ampliado por el que apuestan los "post-translation studies" a los que aludiamos al comienzo de esta investigación, consideramos que una mayor formación traductológica inicial desde un enfoque pragmático contribuiria a que nuestros alumnos comprendieran de forma más específica la relevancia que tiene enmarcar el texto origen en un contexto específico y las soluciones que es necesario adoptar cuando tratamos con aspectos delicados como la traducción de cuestiones de género, asi como la terminología y fraseología relacionada con ello. Esto resulta fundamental para que las opciones de traducción no afecten de forma negativa a la imagen que se proyecta de la construcción de una nueva identidad; máxime si, como en el caso del texto que nos ocupa y las diferentes traducciones analizadas en estas páginas, las identidades que se reescriben se encuentran sometidas a una violencia estructural y simbólica en las sociedades actuales. Por lo tanto, consideramos que exponer a nuestros alumnos a la traducción de este tipo de textos con una formación basada en un enfoque pragmático les permitiría enfrentarse a una nueva realidad cada vez más presente en diversos tipos de tipologias textuales. Asimismo, les ayudaria a desarrollar una mayor concienciación sobre el potencial de su trabajo a la hora de construir y difundir conocimiento y saber mediante el proceso de reescritura que llevan a cabo a través del texto traducido.

\section{REFERENCIAS}

ATIENZA, J. (2017): Los 8 términos que te ayudarán a entender las distintas identidades de género. https://www.lavanguardia.com/vivo/lgtb/20170701/ 423783279046/diccionarioidentidades-de-genero.html (Fecha de consulta $7 / 12 / 2018)$.

AUSTIN, J. L. (1962): How To Do Things With Words. Oxford: Clarendon Press.

BASSNETT, S. (2017): "Foreword" en Gentzler, E. Translation in the Age of Post-Translation Studies. London / New York: Routledge, pp. viii-x.
BROWN, P. \& LEVINSON, S. (1987): Politeness: Some Universals in Language Usage. Cambridge: Cambridge University Press.

COOREN, F. (2008): Between Semiotics and Pragmatics: Opening Language Studies to Textual Agency. Journal of Pragmatics, 40, 1-16.

CORBACHO SÁNCHEZ, A. (2005): "Los conceptos de Tour(ismus) y Reise en los fraseologismos alemanes y su traducción al español". Pragmalingüistica, 13. 6575.

CORPAS PASTOR, G. (ed.) (2000): Las lenguas de Europa: estudios de 
fraseologia, fraseografía y traducción. Granada: Comares.

CORPAS PASTOR, G. (1997): Manual de fraseología española. Madrid: Gredos.

CRONIN, M. (2006): Translation and Identity. London / New York: Routledge.

DICERTO, S. (2018): Multimodal Pragmatics and Translation: A New Model for Source Text Analysis. Cham: Palgrave Macmillan.

FAWCETT, P. (1998): "Presupposition and Translation" en Hickey, L. (ed.) The Pragmatics of Translation. Clevedon: Multilingual Matters, pp. 114-123.

GARCÉS GARCÍA, P. \& FILARDO LLAMAS, L. (2004): "Do Lords think in male?: Gender and Language in Parliamentary Speech". Pragmalingüística, 12. 45-54.

GENTZLER, E. (2012): Translation without Borders. Translation: A Transdisciplinary Journal. Available at http: //translation.fusp.it/articles/translation-without-borders.

GENTZLER, E. (2017): Translation in the Age of Post-Translation Studies. London / New York: Routledge.

GESKE, A. \& GRINFELDS, A. (2006): Education research. Riga: LU.

GRICE, H. P. (1975): 'Logic and conversation', en Cole, P. y Morgan, J. L. (eds.) Syntax and Semantics, vol. 3: Speech Acts, New York: Academic Press, pp. 4158.

GUTT, E. A. (1998): "Pragmatic Aspects of Translation: Some Relevance-Theory Observations" en Hickey, L. (ed.) The Pragmatics of Translation. Clevedon: Multilingual Matters, pp. 4153.

HICKEY, L. (1998): "Introduction" en Hickey, L. (ed.) The Pragmatics of Translation. Clevedon: Multilingual Matters, pp. 1-9.
LEFEVERE, A. \& BASSNETT, S. (1990): "Proust's Grandmother and the Thousand and One Nights" en Bassnett, S. y Lefevere, A. (eds.) Translation, History and Culture. London: Printer Publishers, pp. 1-13.

NATIONAL GEOGRAPHIC (2018): Glosario del Género. https://www.ngenespanol.com /el-mundo/identidad-sexualy-de-genero-definicion-de-identidad-de-genero/

NERGAARD, S. \& ARDUINI, S. (2011): "Translation: A New Paradigm". Translation: A Transdisciplinary Journal 1, 8-17.

ROSKOSA, A. \& RUPNIECE, D. (2016): Advantages and drawbacks of using group work in translator training. Procedia. Social and Behavioral Sciences, No231, pp. 244-250. Disponible en: https://www.sciencedirect.com/science/arti$\mathrm{cle} / \mathrm{pii} / \mathrm{S} 1877042816312095$ (Fecha de consulta: 31/3/18).

SAVENYE, W. C. \& ROBINSON, R. S. (2005): Using qualitative research methods in higher education. Journal of Computing in Higher Education, 16(2), 65-95.

SPERBER, D. \& WILSON, D. (1986): Relevance: Communication and Cognition. Oxford: Blackwell.

STEINMETZ, K. (2017): Beyond 'He' or 'She': The Changing Meaning of Gender and Sexuality. Time. http:/ / time.com/magazine/us/4703292/march27th-2017-vol-189-no-11-u-s/

STEINMETZ, K. (2014): This is What 'Cisgender' means. Time. http://time.com/3636430/cisgender-definition/

SVENSSON, L. \& DOUMAS, K. (2013): Contextual and analytic qualities of research methods exemplified in research on teaching. Qualitative Inquiry. 19. 441-450. 


\begin{abstract}
ANEXO
Encargo: La clínica de Marci L. Bowers (https://marcibowers.com/mtf/) quiere publicar su página web en español para aumentar el número de clientes y te pide la traducción de esta información.

\section{Post-Op Care (GRS) \\ Discharge instructions}

You will be leaving Mills-Peninsula Hospital today. You should be reasonably mobile, able to walk short distances and not in a great deal of pain. About half our patients are off heavy pain medication by the time of discharge. However, you may seek to have wheelchair assistance out to your transportation. Everyone heals at a different pace, so ask for help if you need it.

The DRAIN TUBE is thin but removed on your last day in the hospital just prior to discharge. There remains VAGINAL PACKING soaked with antibiotic gel (Metrogel) inside you and a FOLEY CATHETER for urine drainage. Nursing will meet with you with an actual foley catheter to teach you the finer points of Foley catheter care for the remaining 3 days you will have the thing in your bladder. Your responsibility will be to simply empty the bag when it is full. DON'T BE AFRAID OF YOUR FOLEY-IT IS YOUR FRIEND. If it bothers you, adjust it.

You will be given dilators, lubrication, Neosporin and pads. Apply Neosporin to each incision (on the sides and in the middle) whenever you change your pad-it shouldn't be often, just a few times per day.

\title{
Sexual activity
}

Sexual activity resumption is recommended for those at least 12 weeks out from surgery. Earlier friskiness is possible anecdotally although not endorsed by our office. Orgasm should be an expectation of each and every patient. Keep in mind though that the clitoris is derived from the head of the penis. This area is extremely sensitive and most find it annoyingly so. There can be numbness to the area initially but, again, the arousable areas most likely to lead to an orgasm are probably not the clitoris itself. Orgasm is a complex and complicated combination of sensory inputs and imagination that make it happen. So be inventive and open minded! Each patient of Dr. Bowers will also have the much coveted "B-spot" which is the direct counterpart of the natal female "G-spot". The B-spot is the erectile tissue surrounding the internal urethra which now lies immediately in front of the vaginal wall just beneath the bladder, just as it is in any cis-gender female. Dilating may never be fun for some although with this "b-spot" discovery and its entirely new sensory input, you should find penetration at least interesting. Be patient and persistent! Up to one year may be necessary before rediscovering that 'killer orgasm' although with a female hormonal composition.

Source: https://marcibowers.com/mtf/your-surgery/post-op-care/ (Adapted). 